\title{
Effects of melatonin in experimental stroke models in acute, sub-acute, and chronic stages
}

\author{
Hsiao-Wen Lin \\ E-Jian Lee
}

Neurophysiology Laboratory, Neurosurgical Service, Department of Surgery, National Cheng Kung University Medical Center and Medical School, Tainan, Taiwan
Correspondence: E-Jian Lee Department of Surgery, National Cheng Kung, University Medical Center and Medical School, I 38 Sheng-Li Road, Tainan 70428, Taiwan

Tel +88662353535 ext 5203

Fax +88662766676

Email ejian@mail.ncku.edu.tw

\begin{abstract}
Melatonin (N-acetyl-5-methoxy-tryptamine), a naturally occurring indole produced mainly by the pineal gland, is a well known antioxidant. Stroke (cerebral ischemia) is the second leading cause of death worldwide. To date, however, effective and safe treatment for stroke remains unavailable. Melatonin is both lipid- and water-soluble and readily crosses the blood-brain barrier (BBB). Increasing evidence has shown that, in animal stroke models, administering melatonin significantly reduces infarct volume, edema, and oxidative damage and improves electrophysiological and behavioral performance. Here, we reviewed studies that assess effects of melatonin on cerebral ischemia in acute, sub-acute, and chronic stages. In addition to its potent antioxidant properties, melatonin exerts antiapoptotic, antiexcitotoxic, anti-inflammatory effects and promotes mitochondrial functions in animals with cerebral ischemia. Given that melatonin shows almost no toxicity to humans and possesses multifaceted protective capacity against cerebral ischemia, it is valuable to consider using melatonin in clinical trials on patients suffering from stroke.
\end{abstract}

Keywords: cerebral ischemia, melatonin, stroke, neuroprotection

\section{Introduction}

Stroke (hypoxia/ischemia or ischemia) is the second leading cause of death throughout the world. Focal cerebral ischemia is the major form of stroke and is usually caused by thromboembolic occlusion of supplying arteries. In contrast, global ischemia is often caused by cardiac arrest, massive hemorrhage, or carbon monoxide poisoning. In either circumstance, brain damage occurs when cerebral blood flow drops to $25 \%$ of its normal value. ${ }^{1,2}$ The longer duration of occlusion, the greater damage to the brain and such impairment is usually irreversible. However, limited therapeutic treatments are available clinically and these treatments are only applicable to highly selected groups. For example, tissue plasminogen activator (t-PA), which is the only US Food and Drug Administration (FDA)-approved drug for acute stroke treatment, and prourokinase used for thrombolysis and modified viper venom used for defibrinogenation need to be applied to patients within three to six hours, and it has been noted that these agents increase incidence of intracerebral hemorrhage. ${ }^{3,4}$ Thus, there is an urgent need to have a safe and effective treatment for a larger group of stroke patients.

Reduced blood supply rapidly leads to metabolic failure in the recipient tissues, and such energy shortage directly causes imbalance of ionic gradients across membrane, accumulation of intracellular calcium and sodium ions and reduction of $\mathrm{pH}$. Abnormal ion and $\mathrm{pH}$ gradients disrupt membrane transport, mitochondrial functions and protein production as well as activate $\mathrm{Ca}^{2+}$-dependent enzymatic reaction. As a result of respiratory chain failure, generation of excessive free radicals leads to membrane lipoperoxidation and damages cellular organelles and cytoskeleton. Pro-apoptotic events are initiated, such as activation of nuclear factor $\kappa \mathrm{B}(\mathrm{NF} \kappa \mathrm{B})$ - and $\mathrm{p} 53$-dependent pathways, decreased $\mathrm{Bcl}-2$ to Bax ratio, release of cytochrome c oxidase from mitochondria and caspase activation, and eventually activate DNA-breaking enzymes 
and the energy-consuming DNA repair enzymes resulting in DNA breakdown and cell death. ${ }^{5,6}$ Massive cell death in the brain harms neural network and ultimately impairs neurological functions.

Primary therapeutic strategy in treating cerebral ischemia is to restore blood flow, so that ischemic tissues can be reperfused (or reoxygenated) in the shortest time possible (ideally within minutes) in order to preserve neural tissues, which is also in favor of later functional recovery and survival of individuals. Secondary approach is to ameliorate pathophysiological consequences of stroke, including excitotoxicity, apoptosis, inflammation, penumbral depolarization and diaschisis, caused by free radicals generated during hypoxia/ ischemia and reperfusion. For the former purpose, t-PA and other thrombolytic agents are applied. For the latter, melatonin would be an ideal therapeutic agent since it is a naturally occurring antioxidant, has a known safety profile being virtually nontoxic to humans, ${ }^{7}$ and readily crosses the blood-brain barrier (BBB) when administrated through peripheral routes.

Melatonin (N-acetyl-5-methoxy-tryptamine) is an indole endogenously produced by several mammalian organs, including the pineal gland, retina, and gastrointestinal tract. ${ }^{1}$ In addition to other endogenously produced molecules which showed beneficial effects in animal models of stroke, including estrogen, ${ }^{8}$ erythropoietin ${ }^{9,10,11}$ cannabinoids, ${ }^{12,13}$ melatonin is a potent free radical scavenger and an antioxidant. It can directly detoxify both reactive oxygen, including hydroxyl radical $(\bullet \mathrm{OH}), \mathrm{H}_{2} \mathrm{O}_{2}$ and singlet oxygen $\left({ }^{1} \mathrm{O}_{2}\right)$, and nitrogen species, such as nitric oxide $(\mathrm{NO} \bullet$ ), peroxynitrite anion (ONOO-), and peroxynitrous acid (ONOOH or its activated form, ONOOH•). ${ }^{14}$ Melatonin is also an indirect antioxidant as it can regulate the activities of enzymes which promote overall antioxidative defense systems in an organism, including glutathione peroxidase (GSH-Px), glutathione reductase (GSH-Rd), superoxide dimutase (SOD), and glucose-6-phosphate dehydrogenase (G-6-PD). ${ }^{15}$ In addition, metabolites formed from interaction of melatonin with free radicals, including N1-acetyl-N2-formyl-5-methoxykynuramine (AFMK) and N1-acetyl-5-methoxykynuramine (AMK), are also efficient radical scavengers. ${ }^{16-18}$

Although a considerable number of agents showed neuroprotective effects in animal stroke studies, more than 100 neuroprotectants have failed clinical trials of acute ischemic stroke (AIS). Attention has been drawn recently to modify criteria for evaluating preclinical methods and data due to the latest failed clinical trials on a free radical-trapping agent, NXY-059, in AIS. ${ }^{19}$ Analyses indicated that NXY-059 lacks rigorous testing paradigms in animal models and does not have replicated evidence on its protective effects in extended time window. ${ }^{20-22}$ Suggestions were made that future stroke study needs to be made on more clinical relevant animal models, such as those with other co-morbidity (diabetes, hypertension, etc.). Since stroke is mainly a cardiovascular disease affecting neurological functions, research on the neurovascular system, including neurons, supplying microvessels, endothelium, and glial cells (astrocytes, microglia, and pericytes) will be of great value. Moreover, it is clinically relevant to evaluate effects of a neuroprotectant on the non-ischemic hemisphere and the penumbral regions in the ischemic hemisphere as well as to assess whether it ameliorates complications associated with stroke, including inflammation and electrophysiological and behavioral deficits. This paper aims to analyze available results on the effects of melatonin in animal stroke models at different pathophysiological stages.

\section{Effects of melatonin in acute stroke}

In accordance with the common clinical scenario of stroke, rodent models of focal cerebral ischemia with middle cerebral artery (MCA) occlusion have been widely studied in the laboratory. Temporary occlusion of MCA is performed for 60-90 minutes, and melatonin is administered either before ischemia or shortly after reperfusion. Several physiological parameters, including $\mathrm{pH}, \mathrm{pCO}_{2}, \mathrm{pO}_{2}$, core temperature and blood pressure, are monitored during the course of study and are found unchanged upon administration of melatonin. ${ }^{23,24}$ Abundant literature demonstrated that administering melatonin significantly reduces brain infarction, neurological deficits, and a number of outcome measurement, which has been systemically analyzed and the effect size of melatonin is reported around $0.5 .^{25}$

The protective effects of melatonin in acute stroke (within two days from the onset of vessel occlusion) mainly derives from that melatonin is a direct and indirect antioxidant and that it promotes mitochondrial functions. Antioxidant actions of melatonin include scavenging of free radicals and increasing activity of antioxidant enzymes as described above. Moreover, melatonin suppresses nitric oxide production by reducing nNOS synthesis in neurons in a calmodulin-dependent pathway ${ }^{26}$ and abolishing iNOS expression in macrophages through a NFאB-mediated mechanism. ${ }^{27}$ In addition, melatonin stimulates the activity of NADH-coenzyme Q reductase (Complex I) and cytocrhome c oxidase (Complex IV) in the electron transport chain (ETC) 
of mitochondria and consequently reduces electron leakage and free radical production and maintains normal adenosine triphosphate (ATP) production. ${ }^{28,29}$ This suggests that administering melatonin in stroke could counteract energy shortage to maintain normal cellular function and to decrease brain damage. The ability of melatonin to reduce oxidative damage caused by ischemia has been reported in other organs, such as heart, ${ }^{30}$ liver, ${ }^{31,32}$ and kidney. ${ }^{33}$

Melatonin decreases excitotoxicity and cerebral infiltration of immune cells after cerebral hypoxia/ischemia. Since the pineal gland is the major source of melatonin in the body, pinealectomy was performed on animals to study the effects of endogenous melatonin on cerebral injury. Pinealectomized rats exhibit greater neuronal degeneration and DNA damage in the hippocampus following kainate injection, suggesting that endogenous production of melatonin protects the brain against excitotoxic injury. ${ }^{34}$ Consistent with previous studies with exogenous source of melatonin, pinealectomized rats show larger cortical infarction four or six hours after middle cerebral artery occlusion (MCAO) compared with control animals. Moreover, melatonin is capable of reducing cerebral inflammation after stroke. Administering melatonin at the onset of reperfusion decreases microglial/macrophage activation and reduces immune cell infiltration to the ischemic hemisphere without changing the cellular immune response in the blood stream at two days post-MCAO. ${ }^{35}$ Although such effect could be, at least in part, a result of decreased infarction and other brain damage after melatonin treatment, it has been shown that in an inflammatory rat model of colitis administering melatonin suppresses the mRNA and protein expression of tumor necrosis factor- $\alpha$ (TNF- $\alpha$ ) and intercellular adhesion molecule-1 (ICAM-1) in colon tissues. ${ }^{36}$ Both membrane and nuclear receptors for melatonin have been identified in peripheral blood mononuclear cells, and melatonin stimulation modulates their differentiation, proliferation, and cytokine production, ${ }^{37}$ indicating a direct role of melatonin in controlling immune systems.

Melatonin reduces apoptosis after stroke. Administering melatonin prior to $\mathrm{MCAO}$ or at the onset of reperfusion restores the injury-induced reduction in phosphorylated AKT (p-AKT), $\mathrm{p}$-Bad and Bcl- $\mathrm{X}_{\mathrm{L}}$ levels and the binding of $\mathrm{p}-\mathrm{Bad}$ and 14-3-3 as well as decreases caspase-3 activation. ${ }^{38,39}$ Treatment of melatonin also suppresses the immunoreactivity of cytosolic cytochrome c oxdiase in the ischemic cortex four and 24 hours after MCAO. ${ }^{40}$ To investigate whether melatonin directly inhibits neuronal apoptosis after hypoxia/ ischemia, mouse striatal neurons are cultured and subjected to oxygen and glucose deprivation (OGD). Results showed that melatonin prevents the OGD-induced mitochondrial membrane depolarization and abolishes the NMDA-induced increase in the intracellular $\mathrm{Ca}^{2+}$ levels. It suggests that the antiapoptotic effect of melatonin lies in its inhibition on mitochondrial permeability. Interestingly, as astrocytes have an important role in maintaining brain homeostasis, recent studies demonstrated that melatonin can also reduce glutamate- or $\mathrm{H}_{2} \mathrm{O}_{2}$-induced apoptosis in $\mathrm{C} 6$ astroglial cells. ${ }^{41}$

Melatonin ameliorates brain damage caused by the clinically used thrombolytic agent, t-PA, in animal stroke models. In addition to the widely-studied intraluminal occlusion model of cerebral ischemia, a laser beam-induced occlusion in the cerebral artery has also been invented. In contrast to the use of an Arc beam (which induces fibrin predominantly), the laser beam generates embolism which is similar to the composition of thrombus in humans, ${ }^{42-44}$ making it an ideal model for human stroke. Studies showed that administering melatonin in combination with t-PA at the onset (MCAO) or six hours after reperfusion (laser-beam induced occlusion) decreases t-PA induced iNOS expression and hemorrhagic formation as well as restores p-AKT levels. ${ }^{45}$

Cumulatively, in an acute stage of cerebral hypoxia/ischemia melatonin shows a capacity to reduce brain damage by 1) reducing oxidative stress, 2) promoting mitochondrial functions, 3) decreasing excitotoxicity, 4) suppressing inflammation and 5) diminishing apoptosis.

\section{Effects of melatonin on stroke in sub-acute stage}

Animal studies have been performed to assess the effects of melatonin on stroke in a sub-acute stage (3-14 days). Melatonin-treated animals exhibit improved electrophysiological response compared to vehicle-treated controls at three days post-MCAO. ${ }^{24}$ The somatosensory evoked potentials (SSEPs) are recorded in the left and right primary somatosensory cortex (SI) by stimulating its respective contralateral fore- or hindpaw. The waveforms of the first positive (P1) and the first negative (N1) peaks are used for latency and amplitude measurements. Animals with stroke have severely depressant P1/N1 amplitude in the ischemic forepaw and hindpaw cortical fields but leaving the latency unchanged. Transcallosal diaschisis is observed in the contralateral hindpaw, but not forepaw, cortical field where P1/N1 amplitude is decreased and P1 latency is increased when compared to the pre-ischemic baseline. Administering melatonin reverses the depressant SSEPs in not only the ischemic forepaw and hindpaw cortical fields but also the contralateral hindpaw cortical field. Consistently, 
melatonin-treated animals show improved motor and sensory behavioral scores than vehicle-treated controls.

Similar to brain ischemia, in the spinal cord-injured rats administering melatonin immediately after trauma improves motor and sensory evoked potentials as well as behavioral performance (motor function score and inclined plane test) 10 days after injury. ${ }^{46}$ Biochemical analyses showed that melatonin treatment reduces lipid peroxidation in acute (4 and 24 hour) and sub-acute phases (day 10), compared to untreated injured controls. In summary, these studies show that the neuroprotective effects of melatonin remain significant in a sub-acute phase and that administrating melatonin can effectively prevent electrophysiological and behavioral deficits caused by cerebral hypoxia/ischemia and spinal cord injury.

\section{Long-term effects of melatonin in stroke}

The effects of melatonin after long-term recovery (more than one month) have just been reported lately. Melatonin shows beneficial effects 120 days after global ischemia. ${ }^{47}$ Global ischemia was performed for 15 minutes in rats and melatonin was intravenously administered 30 minutes after the end of ischemia and continued for six hours. Place learning (Morris water maze) and working memory (eight-arm Olton radial maze) was assessed 90 days after ischemia and the pyramidal neurons in cornus Ammoni (CA) region and layers III and V of the medial prefrontal cortex was examined at 120 days post-injury (at the end of behavioral testing). Global ischemia causes a significant delay in acquisition of working memory and impairs place learning as well as reduces pyramidal neurons in the Ammon's horn. As expected, melatonin-treated animals performed significantly better than vehicle-treated controls and similarly to the sham group. In addition, melatonin prevented the ischemia-induced pyramidal neuron loss in the CA regions when compared to vehicle-treated controls, but the remaining neurons in melatonin-treated rats displayed less ramified dendrites than intact controls. In spine structures, melatonin-treated animals showed lower density of spines, mainly composed of mushroom spines, than intact controls. These results suggest that melatonin can counteract the pathophysiological damage caused by ischemia in a long run by preserving neuronal cells and certain degree of the cytoarchitecture of dendrites. Interestingly, it has been shown that melatonin activates $\mathrm{Ca}^{2+}$-dependent alpha isoform of protein kinase $\mathrm{C}$ (PKC) and binds to $\mathrm{Ca}^{2+}$-activated calmodulin to cause microtubule enlargement and neurite outgrowth, and such effect has been implicated in melatonin's preservation of neuronal cytoskeletal organization following brain damage. ${ }^{48,49}$

Furthermore, the role of melatonin in neurogensis has been studied by several different groups but different results were shown. Acute intraperitoneal (ip) injection or chronic treatment of melatonin (in drinking water) was administered on gerbils and the immunoreactivity of doublecortin (Dcx), a marker for newly generated neurons, was examined in the hippocampus 20 weeks after cerebral ischemia. ${ }^{50}$ Results showed that either acute or chronic treatment of melatonin failed to further increase the number of Dcx-positive cells in CA1 regions and decreased the Dcx-positive cells in the dentate gyrus. In the same line, in neural stem cells (NSC) derived from mouse embryo striatum melatonin at pharmacological concentrations (1-100 micromolar) suppressed epidermal growth factor (EGF)-induced NSC proliferation and reduced $1 \%$ fetal bovine serum (FBS)-containing medium-induced neuronal differentiation without affecting astroglial differentiation. ${ }^{51}$ In direct contrast, Kilic and colleagues revealed that delayed (one day after MCAO) and continuous treatment of melatonin (in drinking water) for 29 days increased the number of Dcx-positive cells in the subventricular zone (SVZ) in mice. ${ }^{52}$ It is intriguing whether following cerebral hypoxia/ischemia SVZ and hippocampal stem cells respond differently to chronic treatment of melatonin and whether the difference is a result of a modified stem cell niche after long exposure to melatonin. Further studies are warranted. In the notion that melatonin has anti-inflammatory properties, administering indomethacin, a nonsteroidal anti-inflammatory drug, starting three days before MCAO and continued for 14 or 28 days after ischemia reduces microglial/macrophage activation and increases proliferating cells of all lineage in the cortex and striatum after cerebral hypoxia/ischemia. ${ }^{53}$ It supports a neurogenic hypothesis of melatonin after stroke and suggests a mechanism in which melatonin promotes neurogenesis by suppressing inflammation, and more extensive studies are required to examine these points.

Studies from our lab showed that after MCAO the level of synaptic proteins is significantly higher in the melatonintreated rats compared to vehicle-treated controls; the dendrite density of pyramidal neurons in the ischemic core, penumbra and forelimb and hindlimb somatosensory cortical areas is higher in the melatonin-treated rats than vehicle-treated controls at seven days post-injury (unpublished data). Consistent with the results on melatonin-treated rats three days after MCAO, melatonin improved animals' electrophysiological response at 28 days post-injury (unpublished results). 
Overall, melatonin treatment shows long-term benefits in experimental stroke studies, and data up till today suggest that the effect is a result of better preserved brain tissues in the acute stage.

\section{Concluding remarks}

In addition to its initial protective effects as a free radical scavenger and antioxidant, abundant studies showed that melatonin has multifaceted properties acting against cerebral hypoxia/ischemia and its protection could be long-lasting over months. The outcome measurements in these animal studies, including infarction volume, edema, BBB integrity, electrophysiology, and motor and sensory behaviors, are useful translational parameters and applicable to clinical assessments for the prognosis of stroke patients. Further studies are recommended to evaluate effects of melatonin on stroke animals with other co-morbidity since the physiological background of animals might affect the effectiveness of a treatment and determines whether clinical trials will succeed. Interestingly, gender difference has also been noted in our studies where female animals require lower dose of melatonin to confer neuroprotection after MCAO compared to male counterparts (unpublished observation). It has been shown that estradiol significantly reduces damage caused by cerebral ischemia. ${ }^{54,55}$ It is possible that estrogen and melatonin have synergistic effects and play important roles in determining the severity of cerebral ischemia. This hypothesis requires further investigation. Moreover, neurogenic effects of melatonin remain an important field to explore since it provides important information whether long-term use of melatonin will be helpful for stroke patients.

As melatonin has long been used for ameliorating sleep disturbance and shows no toxicity on humans, clinical trials assessing effects of melatonin on other neurological disorders, including amyotrophic lateral sclerosis (ALS) and Alzheimer's disease, have been conducted. In a group of 31 patients with sporadic ALS, high-dose of melatonin (300 mg/day) given rectally was well tolerated up to two years of observation and it reduced the level of circulating serum protein carbonyls, which is indicative of oxidative stress. ${ }^{56}$ Another double-blind study was conducted on 20 patients with Alzheimer's type of dementia where melatonin (3 mg) was given continuously for four weeks, and results showed that melatonin treatment improved sleep time and night activity as well as cognitive and noncognitive functions. ${ }^{57}$ Similar results were reported by a different group on a larger size of group (45 patients) with longer treatment paradigm (four month period with $6 \mathrm{mg} /$ day).$^{58}$ Data from these clinical trials suggest that administering melatonin is safe and improves neurological functions. In our perspective, administrating melatonin should be done in a timely manner as soon as cerebral ischemia occurs and ideally within three hours. ${ }^{59}$ With its potent antioxidant capacity and other anti-apoptosis and anti-inflammatory effects, melatonin could be therapeutically useful in a clinical setting for patients suffering from cerebral ischemia.

\section{Disclosure}

The authors report no conflicts of interest in this work.

\section{Reference}

1. Reiter RJ, Tan DX, Leon J, Kilic U, Kilic E. When melatonin gets on your nerves: its beneficial actions in experimental models of stroke. Exp Biol Med (Maywood). 2005;230:104-117.

2. Cervantes M, Morali G, Letechipia-Vallejo G. Melatonin and ischemiareperfusion injury of the brain. $J$ Pineal Res. 2008;45:1-7.

3. Tissue plasminogen activator for acute ischemic stroke. The National Institute of Neurological Disorders and Stroke rt-PA Stroke Study Group. N Engl J Med. 1995;333:1581-1587.

4. Furlan A, Higashida R, Wechsler L, et al. Intra-arterial prourokinase for acute ischemic stroke. The PROACT II study: a randomized controlled trial. Prolyse in Acute Cerebral Thromboembolism. JAMA. 1999;282:2003-2011.

5. Lipton P. Ischemic cell death in brain neurons. Physiol Rev. 1999;79:1431-1568.

6. Warner DS, Sheng H, Batinic-Haberle I. Oxidants, antioxidants and the ischemic brain. J Exp Biol. 2004;207:3221-3231.

7. Cheung RT, Tipoe GL, Tam S, Ma ES, Zou LY, Chan PS Preclinical evaluation of pharmacokinetics and safety of melatonin in propylene glycol for intravenous administration. $J$ Pineal Res. 2006;41:337-343.

8. Hurn PD, Macrae IM. Estrogen as a neuroprotectant in stroke. J Cereb Blood Flow Metab. 2000;20:631-652.

9. Sadamoto Y, Igase K, Sakanaka M, et al. Erythropoietin prevents place navigation disability and cortical infarction in rats with permanent occlusion of the middle cerebral artery. Biochem Biophys Res Commun. 1998;253:26-32.

10. Sakanaka M, Wen TC, Matsuda S, et al. In vivo evidence that erythropoietin protects neurons from ischemic damage. Proc Natl Acad Sci US A. 1998;95:4635-4640.

11. Siren AL, Ehrenreich H. Erythropoietin-a novel concept for neuroprotection. Eur Arch Psychiatry Clin Neurosci. 2001;251:179-184.

12. Panikashvili D, Simeonidou $C$, Ben-Shabat $S$, et al. An endogenous cannabinoid (2-AG) is neuroprotective after brain injury. Nature. 2001;413:527-531.

13. Pacher P, Hasko G. Endocannabinoids and cannabinoid receptors in ischaemia-reperfusion injury and preconditioning. BrJ Pharmacol. 2008;153:252-262.

14. Reiter RJ, Tan DX, Manchester LC, Qi W. Biochemical reactivity of melatonin with reactive oxygen and nitrogen species: a review of the evidence. Cell Biochem Biophys. 2001;34:237-256.

15. Reiter R, Tang L, Garcia JJ, et al. Pharmacological actions of melatonin in oxygen radical pathophysiology. Life Sci. 1997;60:2255-2271.

16. Tan DX, Manchester LC, Burkhardt S, et al. N1-acetyl-N2-formyl5-methoxykynuramine, a biogenic amine and melatonin metabolite, functions as a potent antioxidant. FASEB J. 2001;15:2294-2296.

17. Ressmeyer AR, Mayo JC, Zelosko V, et al. Antioxidant properties of the melatonin metabolite N1-acetyl-5-methoxykynuramine (AMK): scavenging of free radicals and prevention of protein destruction. Redox Rep. 2003;8:205-213. 
18. Leon J, Escames G, Rodriguez MI, et al. Inhibition of neuronal nitric oxide synthase activity by N1-acetyl-5-methoxykynuramine, a brain metabolite of melatonin. J Neurochem. 2006;98:2023-2033.

19. Diener HC, Lees KR, Lyden P, et al. NXY-059 for the treatment of acute stroke: pooled analysis of the SAINT I and II Trials. Stroke. 2008;39:1751-1758.

20. Savitz SI. A critical appraisal of the NXY-059 neuroprotection studies for acute stroke: a need for more rigorous testing of neuroprotective agents in animal models of stroke. Exp Neurol. 2007;205:20-25.

21. Savitz SI, Fisher M. Future of neuroprotection for acute stroke: in the aftermath of the SAINT trials. Ann Neurol. 2007;61:396-402.

22. Donnan GA. The 2007 Feinberg lecture: a new road map for neuroprotection. Stroke. 2008;39:242.

23. Pei Z, Pang SF, Cheung RT. Pretreatment with melatonin reduces volume of cerebral infarction in a rat middle cerebral artery occlusion stroke model. J Pineal Res. 2002;32:168-172.

24. Lee EJ, Wu TS, Lee MY, et al. Delayed treatment with melatonin enhances electrophysiological recovery following transient focal cerebral ischemia in rats. J Pineal Res. 2004;36:33-42.

25. Macleod MR, O’Collins T, Horky LL, Howells DW, Donnan GA. Systematic review and meta-analysis of the efficacy of melatonin in experimental stroke. J Pineal Res. 2005;38:35-41.

26. Leon J, Macias M, Escames G, et al. Structure-related inhibition of calmodulin-dependent neuronal nitric-oxide synthase activity by melatonin and synthetic kynurenines. Mol Pharmacol. 2000;58:967-975.

27. Gilad E, Wong HR, Zingarelli B, et al. Melatonin inhibits expression of the inducible isoform of nitric oxide synthase in murine macrophages: role of inhibition of NFkappaB activation. FASEB J. 1998;12:685-693.

28. Acuna-Castroviejo D, Martin M, Macias M, et al. Melatonin, mitochondria, and cellular bioenergetics. $J$ Pineal Res. 2001;30:65-74.

29. Martin M, Macias M, Leon J, et al. Melatonin increases the activity of the oxidative phosphorylation enzymes and the production of ATP in rat brain and liver mitochondria. Int $J$ Biochem Cell Biol. 2002;34:348-357.

30. Reiter RJ, Tan DX. Melatonin: a novel protective agent against oxidative injury of the ischemic/reperfused heart. Cardiovasc Res. 2003;58:10-19.

31. Sewerynek E, Reiter RJ, Melchiorri D, Ortiz GG, Lewinski A. Oxidative damage in the liver induced by ischemia-reperfusion: protection by melatonin. Hepatogastroenterology. 1996;43:898-905.

32. Okatani Y, Wakatsuki A, Reiter RJ, Enzan H, Miyahara Y. Protective effect of melatonin against mitochondrial injury induced by ischemia and reperfusion of rat liver. Eur J Pharmacol. 2003;469:145-152.

33. Sener G, Sehirli AO, Keyer-Uysal M, Arbak S, Ersoy Y, Yegen BC. The protective effect of melatonin on renal ischemia-reperfusion injury in the rat. $J$ Pineal Res. 2002;32:120-126.

34. Manev H, Uz T, Kharlamov A, Joo JY. Increased brain damage after stroke or excitotoxic seizures in melatonin-deficient rats. FASEB J. 1996;10:1546-1551.

35. Lee MY, Kuan YH, Chen HY, et al. Intravenous administration of melatonin reduces the intracerebral cellular inflammatory response following transient focal cerebral ischemia in rats. $J$ Pineal Res. 2007;42:297-309.

36. Li JH, Yu JP, Yu HG, et al. Melatonin reduces inflammatory injury through inhibiting NF-kappaB activation in rats with colitis. Mediators Inflamm. 2005;2005:185-193.

37. Szczepanik M. Melatonin and its influence on immune system. J Physiol Pharmacol. 2007;58(Suppl 6):115-124.

38. Kilic E, Kilic U, Reiter RJ, Bassetti CL, Hermann DM. Tissue-plasminogen activator-induced ischemic brain injury is reversed by melatonin: role of iNOS and Akt. J Pineal Res. 2005;39:151-155.

39. Koh PO. Melatonin attenuates the focal cerebral ischemic injury by inhibiting the dissociation of $\mathrm{pBad}$ from 14-3-3. J Pineal Res. 2008;44:101-106.
40. Andrabi SA, Sayeed I, Siemen D, Wolf G, Horn TF. Direct inhibition of the mitochondrial permeability transition pore: a possible mechanism responsible for anti-apoptotic effects of melatonin. FASEB $J$. 2004;18:869-871.

41. Das A, Belagodu A, Reiter RJ, Ray SK, Banik NL. Cytoprotective effects of melatonin on $\mathrm{C} 6$ astroglial cells exposed to glutamate excitotoxicity and oxidative stress. J Pineal Res. 2008;45:117-124.

42. Watson BD, Prado R, Veloso A, Brunschwig JP, Dietrich WD. Cerebral blood flow restoration and reperfusion injury after ultraviolet laserfacilitated middle cerebral artery recanalization in rat thrombotic stroke. Stroke. 2002;33:428-434.

43. Wysokinski WE, Owen WG, Fass DN, Patrzalek DD, Murphy L, McBane RD 2nd. Atrial fibrillation and thrombosis: immunohistochemical differences between in situ and embolized thrombi. J Thromb Haemost. 2004;2:1637-1644.

44. Marder VJ, Chute DJ, Starkman S, et al. Analysis of thrombi retrieved from cerebral arteries of patients with acute ischemic stroke. Stroke. 2006;37:2086-2093.

45. Chen TY, Lee MY, Chen HY, et al. Melatonin attenuates the postischemic increase in blood-brain barrier permeability and decreases hemorrhagic transformation of tissue-plasminogen activator therapy following ischemic stroke in mice. J Pineal Res. 2006;40:242-250.

46. Cayli SR, Kocak A, Yilmaz U, et al. Effect of combined treatment with melatonin and methylprednisolone on neurological recovery after experimental spinal cord injury. Eur Spine J. 2004;13:724-732.

47. Letechipia-Vallejo G, Lopez-Loeza E, Espinoza-Gonzalez V, et al. Long-term morphological and functional evaluation of the neuroprotective effects of post-ischemic treatment with melatonin in rats. $J$ Pineal Res. 2007;42:138-146.

48. Huerto-Delgadillo L, Anton-Tay F, Benitez-King G. Effects of melatonin on microtubule assembly depend on hormone concentration: role of melatonin as a calmodulin antagonist. J Pineal Res. 1994;17:55-62.

49. Benitez-King G, Hernandez ME, Tovar R, Ramirez G. Melatonin activates PKC-alpha but not PKC-epsilon in N1E-115 cells. Neurochem Int. 2001;39:95-102.

50. Rennie K, de Butte M, Frechette M, Pappas BA. Chronic and acute melatonin effects in gerbil global forebrain ischemia: long-term neural and behavioral outcome. J Pineal Res. 2008;44:149-156.

51. Moriya T, Horie N, Mitome M, Shinohara K. Melatonin influences the proliferative and differentiative activity of neural stem cells. J Pineal Res. 2007;42:411-418.

52. Kilic E, Kilic U, Bacigaluppi M, et al. Delayed melatonin administration promotes neuronal survival, neurogenesis and motor recovery, and attenuates hyperactivity and anxiety after mild focal cerebral ischemia in mice. J Pineal Res. 2008;45:142-148.

53. Hoehn BD, Palmer TD, Steinberg GK. Neurogenesis in rats after focal cerebral ischemia is enhanced by indomethacin. Stroke. 2005;36:2718-2724.

54. Toung TJ, Traystman RJ, Hurn PD. Estrogen-mediated neuroprotection after experimental stroke in male rats. Stroke. 1998;29:1666-1670.

55. Green PS, Yang SH, Nilsson KR, Kumar AS, Covey DF, Simpkins JW. The nonfeminizing enantiomer of 17 beta-estradiol exerts protective effects in neuronal cultures and a rat model of cerebral ischemia. Endocrinology. 2001;142:400-406.

56. Weishaupt JH, Bartels C, Polking E, et al. Reduced oxidative damage in ALS by high-dose enteral melatonin treatment. $J$ Pineal Res. 2006;41:313-323.

57. Asayama K, Yamadera H, Ito T, Suzuki H, Kudo Y, Endo S. Double blind study of melatonin effects on the sleep-wake rhythm, cognitive and non-cognitive functions in Alzheimer type dementia. J Nippon Med Sch. 2003;70:334-341.

58. Cardinali DP, Brusco LI, Liberczuk C, Furio AM. The use of melatonin in Alzheimer's disease. Neuro Endocrinol Lett. 2002;23(Suppl 1):20-23.

59. Pei Z, Pang SF, Cheung RT. Administration of melatonin after onset of ischemia reduces the volume of cerebral infarction in a rat middle cerebral artery occlusion stroke model. Stroke. 2003;34:770-775. 